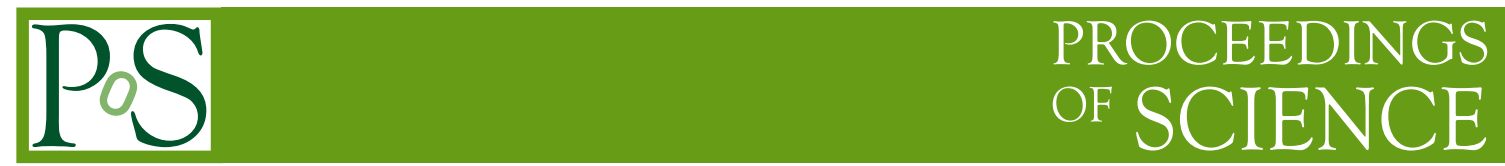

\title{
Measured Cosmogenic Background at RENO
}

\section{Daeun Jung*}

Sungkyunkwan University

E-mail: cowalker12@gmail.com

On behalf of the RENO collaboration

\begin{abstract}
The isotopes of ${ }^{9} \mathrm{Li}$ and ${ }^{8} \mathrm{He}$ produced by cosmic-rays are one of main source for backgrounds in reactor neutrino experiments. The isotope decays to a neutron and an electron and mimics an inverse beta decay of an electron antineutrino from reactors. The ${ }^{9} \mathrm{Li} /{ }^{8} \mathrm{He}$ background spectrum and rate are measured using the data taken by the RENO, and compared with Monte-Carlo prediction. In this presentation, we report the measured cosmogenic background spectrum and rate at RENO.
\end{abstract}

The 39th International Conference on High Energy Physics (ICHEP2018)

4-11 July, 2018

Seoul, Korea

${ }^{*}$ Speaker. 


\section{Introduction}

Detection of a neutron and a positron is the distinctive feature of Inverse Beta Decay (IBD) events which occur through the interaction between an electron antineutrino and a proton. A positron is promptly annihilated with an electron emitting two photons while a neutron is captured by gadolinium emitting photons after a few multiple scatterings with nucleus. Cosmic muons interact with carbon nucleus producing unstable ${ }^{9} \mathrm{Li}$ and ${ }^{8} \mathrm{He}$. These isotopes decay to a neutron and an electron which can mimic an IBD event. In the RENO experiment ${ }^{9} \mathrm{Li} /{ }^{8} \mathrm{He}$ isotopes contributes to a significant portion of backgrounds so that understanding of ${ }^{9} \mathrm{Li} /{ }^{8} \mathrm{He}$ energy spectra and rates is very important for neutrino oscillation measurements. In this paper, the measured ${ }^{9} \mathrm{Li} /{ }^{8} \mathrm{He}$ spectra from the RENO data are compared with Monte-Carlo (MC) predictions.

\section{Monte-Carlo simulation}

Using the GEANT 4 based detector simulations, the energy spectra are generated for each decay mode of the isotopes and the total energy spectra are obtained by combining these spectra with decay probabilities. Detector geometry, material, and PMT characteristics are implemented in the simulation and the identical IBD selection cuts as data are applied. The number of photoelectrons from PMTs are converted to the neutrino energy using the detector response to ${ }^{137} \mathrm{Cs}$, ${ }^{68} \mathrm{Ge},{ }^{60} \mathrm{Co}$, and neutron capture events by hydrogen, carbon, and gadolinium [1]. An additional energy resolution is applied to Monte-Carlo sample to compensate for the difference between data and Monte-Carlo as shown in Figure 1.

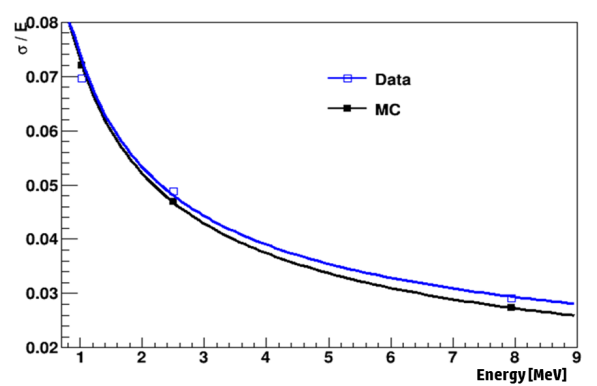

Figure 1: Energy resolution as a function of energy

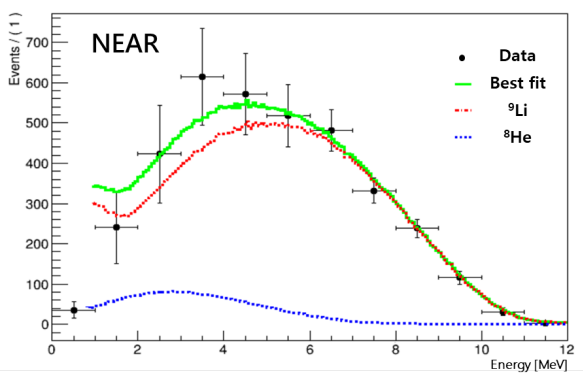

Figure 2: ${ }^{9} \mathrm{Li}$ and ${ }^{8} \mathrm{He}$ energy spectrum at near detector

\section{Result}

Monte-Carlo beta spectra of ${ }^{9} \mathrm{Li}$ and ${ }^{8} \mathrm{He}$ are fitted to the observed spectra from 2200 days of RENO data. It is shown that in near(far) detector, the fraction of ${ }^{9} \mathrm{Li}$ component is $91.6 \pm 2.3(100 \pm$ 8.7)\%. ${ }^{9} \mathrm{Li}$ background is found to be dominant in both detectors as shown in Figure 2.

\section{References}

[1] W. Choi, Observation of Energy and Baseline Dependent Reactor Neutrino Disappearance in the RENO Experiment, Ph.D Thesis, Seoul National Universiy, 2016. 